\title{
Age-Related Histological Changes in Kidneys of Brown Norway Rat
}

\author{
Akira YABUKI ${ }^{1) *}$, Shinichiro YONESHIGE ${ }^{1)}$, Shin TANAKA $^{2)}$, Masashi TSUJIO ${ }^{3)}$, Sawane MITANI ${ }^{1)}$ and \\ Osamu YAMATO ${ }^{1)}$ \\ 1) Laboratory of Veterinary Clinical Pathology, Joint Faculty of Veterinary Medicine, Kagoshima University, 1-21-24 Korimoto, \\ Kagoshima 890-0065, Japan \\ ${ }^{2)}$ Formerly National Center for Geriatrics and Gerontology (NCGG), 35 Gengo, Morioka-machi, Obu, Aichi 474-8511, Japan \\ ${ }^{3)}$ Laboratory of Veterinary Anatomy, Joint Faculty of Veterinary Medicine, Kagoshima University, 1-21-24 Korimoto, Kagoshima \\ 890-0065, Japan
}

(Received 23 August 2013/Accepted 25 September 2013/Published online in J-STAGE 9 October 2013)

ABSTRACT. In this study, age-dependent histological changes in the kidneys of Brown Norway rat, a strain useful for conducting aging research, were evaluated. Examination was performed at 3, 12, 18, 24 and 30 months of age. Sclerotic and hypertrophic changes of the glomeruli were observed, and quantitative scores of these changes persistently increased with age. A marginal increase in scores was observed for glomerular cystic changes and tubulointerstitial damage. Further, urothelial hyperplasia was observed in the renal papillae, particularly at 30 months of age. In conclusion, the findings of the present study demonstrate that the Brown Norway strain exhibits persistent, but mild progression of age-dependent renal histological changes.

KEY WORDS: aging, Brown Norway rat, glomerulus, histological change, kidney.

doi: 10.1292/jvms.13-0431; J. Vet. Med. Sci. 76(2): 277-280, 2014

Chronic kidney disease (CKD) is a global health problem especially in the elderly, and a large number of aged patients are diagnosed during the early stages of CKD [3, 7]. The Brown Norway (BN) rat is an inbred strain with longevity and is suitable for use in aging studies $[5,12]$. Age-dependent renal damages in $\mathrm{BN}$ rats was previously assessed and compared with those in other inbred rats, and it was found that chronic nephrosis progressed more slowly in $\mathrm{BN}$ rats [6]. This slow progression is useful for modeling age-dependent, naturally occurring CKD, and clarifying age-dependent histological changes in the kidneys of $\mathrm{BN}$ rats is useful for understanding the pathological mechanisms of elderly CKD. However, renal histological changes related to natural aging of $\mathrm{BN}$ rats have not been thoroughly investigated. In the present study, age-dependent changes in $\mathrm{BN}$ rat kidneys were analyzed histomorphometrically.

All experimentation in the present study proceeded in accordance with the Guidelines for Animal Experimentation of the National Center for Geriatrics and Gerontology (NCGG), Japan. Male BN/CrlCrlj rats aged 3, 12, 18, 24 and 30 months ( $\mathrm{n}=5$; per age group) were used in the present study. The rats were housed in temperature- and humiditycontrolled animal rooms at the National Center for Geriatrics and Gerontology (Obu, Japan) [15] and fed a commercial diet (Labo MR Stock; Nihon Nousan, Yokohama, Japan).

\footnotetext{
*Correspondence to: Yabuki, A., Laboratory of Veterinary Clinical Pathology, Joint Faculty of Veterinary Medicine, Kagoshima University, 1-21-24 Korimoto, Kagoshima 890-0065, Japan. e-mail: yabu@vet.kagoshima-u.ac.jp

(C)2014 The Japanese Society of Veterinary Science

This is an open-access article distributed under the terms of the Creative Commons Attribution Non-Commercial No Derivatives (by-nc-nd) License $<$ http://creativecommons.org/licenses/by-nc-nd/3.0/>.
}

Water was supplied ad libitum. All rats exhibited no apparent pathological changes in their appearance, except for tarsal tylosis of aged ones. They were sacrificed by exsanguination under deep anesthesia using a mixture of 2, 2, 2-tribromoethanol and 2-methyl-2butanol (Avertin; $300 \mathrm{mg} / \mathrm{kg}$, i.p.). Kidneys were quickly removed, cut perpendicularly to the long axis and fixed in 10\% neutral-buffered formalin. After routine paraffin embedding, $3-\mu \mathrm{m}$ thick sections were cut, and sections were selected every $30 \mu \mathrm{m}$ and stained with hematoxylin-eosin (HE), periodic acid Schiff (PAS) and Masson's trichrome stains. Following parameters were assessed using 3 sections from each animal. 1) Diameter of renal corpuscle [16], 2) percentage of cystic renal corpuscles; $100 \times$ (number of cystic renal corpuscles / total number of glomeruli), 3) extent of glomerular sclerotic change [16], 4) extent of interstitial fibrosis [16] and 5) extent of interstitial mononuclear cell infiltration [16]. Blood samples were able to be collected from animals aged 18, 24 and 30 months. Plasma (heparinized) was obtained after centrifugation and stored at $-20^{\circ} \mathrm{C}$. The concentration of blood urea nitrogen (BUN) was measured using a spectrophotometer and a test kit (Nitrogen B-test kit, Wako Pure Chemical, Osaka, Japan), and blood creatinine and electrolyte ( $\mathrm{Na}, \mathrm{K}$ and $\mathrm{Cl}$ ) levels were measured using a dry-chemistry analyzer (DRICHEM 7000V, Fujifilm, Tokyo, Japan). Statistical analysis was performed using PASW software (IBM SPSS Statistics, Armonk, NY, U.S.A.). The Kruskal-Wallis test was used to evaluate age-dependent changes $(P<0.05)$.

In the renal corpuscles, glomerular sclerotic changes characterized by expansion of mesangial matrices, proliferation of mesangial cells and thickening and wrinkling of the glomerular basement membrane (GBM) were observed in aged rats (Fig. 1A and 1B). Cystic changes were also observed in the renal corpuscles (Fig. 1C). Glomerular tufts in 



Fig. 1. Light microscopy images of BN rat kidneys. A) Cortex of a 3-month-old rat. The left glomerulus showed severe sclerotic change, but the right glomerulus had a normal structure. Masson's trichrome (MT) stain. Bar: $20 \mu \mathrm{m}$. B) Cortex of a 30-month-old rat. A glomerulus with severe sclerosis and hypertrophy. MT stain. Bar: $20 \mu \mathrm{m}$. C) Cortex of a 12-month-old rat. *: Cystic changes of renal corpuscles. Periodic acid Schiff stain. Bar: $50 \mu \mathrm{m}$. D) Cortex of a 30-month-old rat. Damaged tubules, interstitial fibrosis and interstitial mononuclear cell infiltration (arrow) were observed, but the severity of this damage was relatively mild. MT stain. Bar: $100 \mu \mathrm{m}$. E) Renal papilla of a 12-month-old rat. Mild hyperplastic change of the urothelium is observed in the marginal area of the basal portion of the papilla (arrow). Papi: papilla. US: urinary space. Hematoxylin-eosin (HE) stain. Bar: $200 \mu \mathrm{m}$. F) Renal papilla of a 30-month-old rat. Hyperplastic urothelium is seen in the wide marginal area (arrows). **: Hematomas. HE stain. Bar: $200 \mu \mathrm{m}$.

the cystic renal corpuscles were relatively well preserved, although some glomeruli showed sclerotic changes. Tubular atrophy, interstitial mononuclear cell infiltration and interstitial fibrosis were observed in aged rats, although the severity of these damages was mild even in rats aged 30 months (Fig. 1D). Hyperplasia of the urothelial layer was observed in areas where it directly covered the papillae or medulla within the renal sinus. These hyperplastic lesions were observed in
1 animal aged 12 months, 1 animal aged 18 months and 1 animal aged 24 months (Fig. 1E). In 30 months, all animals showed relatively severe lesions (Fig. 1F). Hematomas were occasionally observed concomitantly.

The diameter of the renal corpuscle and the score for glomerulosclerosis continuously increased from 3 to 30 months of age (Fig. 2A and 2B). The percentage of cystic glomeruli increased between 3 and 12 months, but additional elevations 

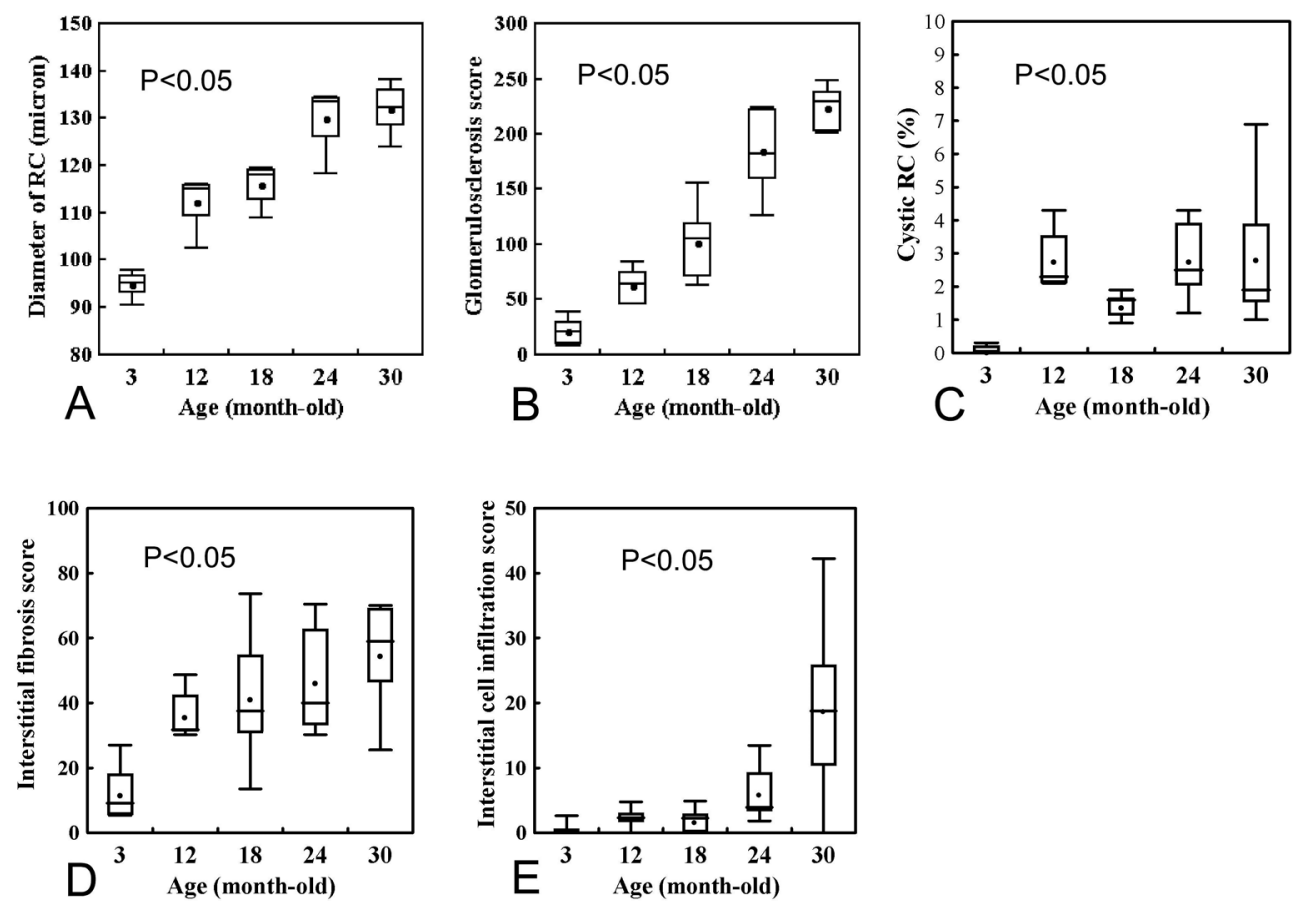

Fig. 2. Results of the quantitative analysis. A) Renal corpuscle diameter. B) Glomerulosclerosis extent. C) Percentage of cystic renal corpuscles. D) Interstitial fibrosis extent. E) Extent of interstitial mononuclear cell infiltration. Data are shown in box plots. RC: renal corpuscle.

were not detected after 12 months (Fig. 2C). The interstitial fibrosis score showed a mild increase from 3 to 12 months (Fig. 2D). While continuous increases were observed after 12 months, these increases were very mild. Mononuclear cell infiltration score did not increase until 24 months, but slight increases were observed between 24 and 30 months (Fig. 2E). Changes in these parameters were statistically significant. No significant changes were observed in the blood chemical analysis (Table 1).

Glomeruloscrelosis, glomerular hypertrophy and interstitial fibrosis are known as the age-dependent histological changes in the rat kidneys [17], and $\mathrm{BN}$ rats also showed these changes. Especially, prominent changes were observed in the glomeruli. Quantitative analysis showed that both the glomerulosclerosis score and renal corpuscle diameter continually increased between 3 and 30 months of age, suggesting a close association between glomerulosclerosis and glomerular hypertrophy. Since progression of glomerulosclerosis induces a reduction of functional nephron number, glomerular capillary hydraulic pressure in the remnant functional glomeruli increased in accordance with Brenner's hyperfiltration theory [1]. This adaptational hyperfunction in the remnant glomeruli induced subsequent glomerular hypertrophy. However, glomerular hypertrophy and sclerosis may have been induced due to age-dependent complex physiological changes, such as oxidative stress [13] and hypertension, among other reasons [4].

Cystic changes were observed in the renal corpuscles and were considered to be a distinct change not associated
Table 1. Results of the blood chemistry tests

\begin{tabular}{lrrr}
\hline & 18 months & 24 months & 30 months \\
\hline BUN $(\mathrm{mmol} / l)$ & $8.1(0.6)$ & $8.1(0.9)$ & $8.5(1.0)$ \\
Creatinine $(\mu \mathrm{mol} / l)$ & $26.5(4.4)$ & $26.5(7.1)$ & $17.7(2.7)$ \\
Sodium $(\mathrm{mmol} / l)$ & $145(1.7)$ & $146(1.3)$ & $146(0.8)$ \\
Potassium $(\mathrm{mmol} / l)$ & $5.1(0.3)$ & $5.1(0.3)$ & $5.1(0.6)$ \\
Chloride $(\mathrm{mmol} / l)$ & $104(1.0)$ & $103(1.5)$ & $102(2.0)$ \\
\hline
\end{tabular}

All data represent the median (interquartile deviation). No significant differences were observed for all parameters $(P<0.05)$. BUN: blood urea nitrogen.

with the glomerulosclerosis and glomerular hypertrophy. Although cystic changes of the renal corpuscle have wellknown histological features similar to autosomal polycystic kidney disease and renal dysplasia [10], these congenital diseases were not suspected in the present study, and it was difficult to determine the cause of cystic changes of the renal corpuscles in $\mathrm{BN}$ rats.

Hyperplastic urothelium was previously reported in BN rats [14], however, age-dependent changes have remained unclear. In the present study, urothelial hyperplasia in the renal papillae was shown to be an age-dependent pathological feature in the kidneys of BN rats. In clinically, hyperplasia of the urothelium has been reported in humans treated with phenacetin, which is an analgesic drug that shows adverse effects during urothelial carcinoma [2]. However, no malignant findings were observed in the present study, and the clinical significance of this urothelial lesion in the $\mathrm{BN}$ rats 
remained unclear.

Tubulointerstitial damage (TID) has attracted attention as a common pathway towards end-stage renal failure in CKD patients $[8,11]$. In the present study, although quantitative scores for interstitial cell infiltration and interstitial fibrosis, which are histological hallmarks of TID, increased slightly, we identified TID as a minor renal lesion even in senile 30-month-old rats. It is well known that TID is not an initial renal pathological feature of naturally occurring CKD, which substantially progresses when renal damage reaches a threshold state $[8,11]$. Since glomerular injury is the initial pathological change indicating CKD $[8,9]$, glomerular damages of $\mathrm{BN}$ rats may not have reached the threshold level to induce TID progression until 30 months of age. Indeed, blood chemical tests showed no significant changes of BUN, creatinine and electrolytes concentrations until 30 months of age. In a previous study, the $\mathrm{BN}$ rat showed slow progression of age-dependent chronic nephrosis, and the degree of nephrosis maintained at a minimal level until the terminal life stage [6]. However, why nephrosis progression is delayed in $\mathrm{BN}$ rats has remained unclear. In the present study, we suspected that persistent progression of glomerulosclerosis; thus, slow and minimal progression of TID might be associated with the slow progression of nephrosis in $\mathrm{BN}$ rats.

\section{REFERENCES}

1. Brenner, B. M., Lawler, E. V. and Mackenzie, H. S. 1996. The hyperfiltration theory: a paradigm shift in nephrology. Kidney Int. 49: 1774-1777. [Medline] [CrossRef]

2. Bringuier, P. P., McCredie, M., Sauter, G., Bilous, M., Stewart, J., Mihatsch, M. J., Kleihues, P. and Ohgaki, H. 1998. Carcinomas of the renal pelvis associated with smoking and phenacetin abuse: p53 mutations and polymorphism of carcinogenmetabolising enzymes. Int. J. Cancer 79: 531-536. [Medline] [CrossRef]

3. Coresh, J., Selvin, E., Stevens, L. A., Manzi, J., Kusek, J. W., Eggers, P., Van Lente, F. and Levey, A. S. 2007. Prevalence of chronic kidney disease in the United States. JAMA 298: 20382047. [Medline] [CrossRef]

4. Duarte, D., Santos-Araújo, C. and Leite-Moreira, A. F. 2011. Hypertension and angiogenesis in the aging kidney: a review. Arch. Gerontol. Geriatr. 52: e93-e102. [Medline] [CrossRef]

5. Gordon, C. J., Rowsey, P. J., Bishop, B. L., Ward, W. O. and Macphail, R. C. 2011. Serum biomarkers of aging in the Brown
Norway rat. Exp. Gerontol. 46: 953-957. [Medline] [CrossRef]

6. Gray, J. E., van Zwieten, M. J. and Hollander, C. F. 1982. Early light microscopic changes in chronic progressive nephrosis in several strains of aging laboratory rats. J. Gerontol. 37: 142-150. [Medline] [CrossRef]

7. Hallan, S. I., Coresh, J., Astor, B. C., Asberg, A., Powe, N. R., Romundstad, S., Hallan, H. A., Lydersen, S. and Holmen, J. 2006. International comparison of the relationship of chronic kidney disease prevalence and ESRD risk. J. Am. Soc. Nephrol. 17: 2275-2284. [Medline] [CrossRef]

8. Hodgkins, K. S. and Schnaper, H. W. 2012. Tubulointerstitial injury and the progression of chronic kidney disease. Pediatr. Nephrol. 27: 901-909. [Medline] [CrossRef]

9. Kriz, W. and LeHir, M. 2005. Pathways to nephron loss starting from glomerular diseases-insights from animal models. Kidney Int. 67: 404-419. [Medline] [CrossRef]

10. Liapis, H. and Winyard, P. 2007. Cystic diseases and developmental kidney defects. pp. 1257-1306. In: Heptinstall's Pathology of the Kidney, 6th ed. (Jennette, J. C., Olson, J. L., Schwartz, M. M. and Silva, F. G. eds.), Lippincott Williams \& Wilkins, Philadelphia.

11. Nangaku, M. 2006. Chronic hypoxia and tubulointerstitial injury: a final common pathway to end-stage renal failure. J. Am. Soc. Nephrol. 17: 17-25. [Medline] [CrossRef]

12. Phillips, P. M., Jarema, K. A., Kurtz, D. M. and MacPhail, R. C. 2010. An observational assessment method for aging laboratory rats. J. Am. Assoc. Lab. Anim. Sci. 49: 792-799. [Medline]

13. Rice, K. M., Preston, D. L., Walker, E. M. and Blough, E. R. 2006. Aging influences multiple incidices of oxidative stress in the aortic media of the Fischer 344/NNiaxBrown Norway/BiNia rat. Free Radic. Res. 40: 185-197. [Medline] [CrossRef]

14. Stub, C., Thon, R., Ritskes-Hoitinga, M. and Hansen, A. K. 2004. Renal epithelial proliferation and its clinical expression in Brown Norway (BN) rats. Lab. Anim. 38: 85-91. [Medline] [CrossRef]

15. Tanaka, S., Segawa, T., Tamaya, N. and Ohno, T. 2000. Establishment of an aging farm of F344/N rats and C57BL/6 mice at National Institute for Longevity Sciences (NILS). Arch. Gerontol. Geriatr. 30: 215-223. [Medline] [CrossRef]

16. Yabuki, A., Mitani, S., Sawa, M., Mizukami, K., Fujiki, M. and Yamato, O. 2012. A comparative study of chronic kidney disease in dogs and cats: induction of cyclooxygenases. Res. Vet. Sci. 93: 892-897. [Medline] [CrossRef]

17. Yabuki, A., Tanaka, S., Matsumoto, M., Kamimura, R. and Suzuki, S. 2004. Age-related histological changes in kidneys of female F344/N rats. Kyushu J. Exp. Anim. 20: 9-14 (in Japanese with English abstract). 